\title{
Measuring the Performance of Jordanian Islamic Banks
}

\author{
Yazan Qasim (Corresponding author) \\ Faculty of Economics and Muamalat, Universiti Sains Islam Malaysia \\ 71800 NilaiNegeri Sembilan, Malaysia \\ Email: wolf_mgableh@yahoo.com
}

\author{
Yazis Mohamad \\ Faculty of Economics and Muamalat, Universiti Sains Islam Malaysia \\ 71800 NilaiNegeri Sembilan, Malaysia \\ Email: yazis@usim.edu.my
}

Norhazlina Ibrahim

Faculty of Economics and Muamalat, Universiti Sains Islam Malaysia

71800 NilaiNegeri Sembilan, Malaysia

Email: norhazlina@usim.edu.my

Received: January 09, 2017 Accepted: February 08, 2017 Published: February 18, 2017

doi:10.5296/jpag.v7i1.10575 URL: http://dx.doi.org/10.5296/jpag.v7i1.10575

\begin{abstract}
The banking sector is believed to be one of the driving forces of economic growth of many countries. Muslim jurists have realized the need to get the benefit of banking activities that adopts a lawful way with Islamic rule. Jordanian Islamic banks suffer from measure the financial performance using effective measurement tool based on various indicators. The main aim of this research is to analysis and ranks the performance levels of the Jordanian Islamic banks using suitable measurement methods. This study utilizes secondary data to measures the performance of three Jordanian Islamic banks (JIBFI, IIAB, and JDIB) over the period 2010-2013 by integrated tools; FRA, DEA, and MI. The significant results indicate that JDIB recorded the best performance rank based the three measurements tools, followed IIAB, and lowest rank is JIBFI. The contribution of this study is performance measurements of Jordanian Islamic banks based on the combination of FRA, DEA, and MI rather than
\end{abstract}


utilize one measurement tool.

Keywords: Jordanian Islamic banks, Performance measurement, FRA, DEA, MI.

\section{Introduction}

Nowadays, the banking sector is believed to be one of the driving forces of economic growth of many countries. They are also regarded as the backbone of any economy because of their roles in regulating and facilitating finances to individuals, industry, and social institutions (Assi, 2010). Banks play an important role in the domestic and international economy through their dual roles in the inter-mediation of excess liquidity accepting them in form of deposits savings and subsequently uses a great percentage of these deposits in the form of credit facilities, investments, and loans to allow funding for economic activities (Hamouri et al., 2014). Islamic Banks operate a system that derived from the principles of Islamic Shariah law and governed by the concepts of Islamic economics (Al-Talib, 2005). In Jordan there are three Islamic bank; the first one that was established in 1978 was called Jordan Islamic bank for finance and investment followed by the Islamic international Arab bank created in 1997 and recently the Jordanian Dubai Islamic bank created in 2010 (Al-Naimat, 2013).

The performance measurement considered as an essential need for the bank's growth and to determine the best solutions for the problems that faced this bank (Kurd, 2010). There are several banking studies conducted for measuring the financial performance by using several measurement tools to examine the relationship between banks and financial performance. The most known performance measurement tools of Jordanian Islamic banks are: 1) Financial Ratio Analysis (FRA). 2) Data Envelopment Analysis (DEA). 3) Maqasid Index (MI). These tools are used to measure the profitability, costs, and efficiency of Islamic banks. However, Jordanian Islamic Banks were mostly utilized one measurement method rather than two or three methods as combination of performance measurement. This research measures the performance and compare between Jordanian IBs based on combination of three analytical tools; FRA, DEA, and MI, in order to enhance the usefulness of performance measurement.

Bank's performance is important for all parties; depositors, bank managers, and regulators. Also, in the competitive market, bank performance provides a signal to investors-depositor whether to withdraw funds invest or withdraw funds from the bank. Then the improved the services (loan or deposit) or both of it to improve its finance (Samadet al., 1999). Performance measurement is considered as an essential need for growth and prosperity. In addition to maintaining the growing and sustainability of the Islamic banks, financial performance, and efficiency have to be measured regularly (Kurd, 2010). The main objective of this study is to utilize three integrated performance measurement tools; FRA, DEA, and MI. In order to increases the measurement accuracy of banks performance.

\section{Literature review}

During the study of literature review, the researcher asserts to some of the results although, FRA technique utilized to measure performance efficiency of Islamic banks. We need to use 
FRA for determining the financial situations of Islamic banks (Johnes\& et al., 2009). In relation to the DEA technique, it appears fewer advances have been made to the FRA technique and its utilization begins in 1984 as a new technique to measure the performance. Also, DEA utilized to control costs and generating more profits (Omari, 2013). However, it can be observed that Maqasidshariah's index is to choose a solution from alternatives candidates that lead to an improvement in decision making (Antonio \& et al., 2012). It is a new analytical tool that will be adapted to investigate performance efficiency of Jordanian Islamic banks.

There are previous studies about the measurement of a financial performance conducted around the world. Recently, conducted study by Hunjra et al. (2014) in Pakistan to measure the financial performance between the Conventional Banks (CBs) and Islamic Banks (IBs) in order to assist all the bank shareholders, managers, depositors, regulators and investors by providing true details of the financial position using ratio analysis of their annual financial of the years 2008-2012 period. Nineteen ratios were used to investigate these performances in terms of capital adequacy, profitability, risk and solvency, liquidity, operational, deployment and cash flow and cash flow ratio. The finding resolves that IBs are less profitable, deployed and operationally competent with more liquid and less risky investment in comparison with CBs. There are significant mean differences at the profitability, capital adequacy, and cash flow ratio on these banks.

Shehzadet al. (2012) performed a study conducted in Pakistan with the intention of making the comparison between the financial performances of the Islamic banking sector with CBs during the period 2007-2011. To present a vivid picture of IB to the stakeholders, the financial analysis of IBs has not been thorough as compared to well-established CBs. To investigate the performance, six FRA were useful in testing the performance of 8 sampled banks. The finding shows that IBs are less risky and less competent in Expense Management (EM) when it is likened with the CBs.

According to Najjar (2013), an investigation of the financial performance of Al-Bahrain banks is covering the estimation of essential financial ratios of major financial institutions compares its performance in the context of the worldwide crisis in 2008. It makes a comparison of ratios between the $\mathrm{CBs}$ and Islamic financial institutions. These ratios describe the financial performance, profitability, type of banks and size. These investigation ratios reveal the changing in the financial management practices of banks in their individual areas. Also, findings show the existence of broad divergence in the ratios utilized by IBs and CBs particularly prior to the financial crisis and subsequently, as well as it helps to clarify the finest practice in the areas of the liquidity management, profitability management, interest rate and risk management.

Also, Johns, Izzeldin \& Pappas (2009) accomplished their research by comparing the performance of interest and non-interest based banks using the DEA after the financial crisis and during the crisis of 2004-2009. Non-Islamic and IBs showed an insignificant dissimilarity in mean when efficiency is measured against the common frontier. However, there was a few of basic change while using the Meta Frontier Analysis (MFA) between the 
IBs and CBs. The IBs efficiency frontier lies inside the frontier for CB. This location inside the frontier reveals that the IBs system is less competent during the comparison to CBs. Johnes, Izzeldin \& Pappas (2012); Majid (2005) and Bhattacharyya et al. (2010) found an amount of reasons for estimating the lower performance of IBs than Non-Islamic banks (N-IB). First of all, because of stringent Shariah rules and restrictions which limits products are not being standardized and then the consequence is enhanced operational cost in comparison to N-IB. Secondly, a size of the IB is small in the examination with non-Islamic banks with an increased technical efficiency (Majid et al. 2005; Bhattacharyya et al., 2010).

A review of analyzing the Islamic banks' efficiency using both an alternative approach and standard approach found that the incompetence level among Islamic banks using the Standard Data Envelopment Approach (S-DEA) is greater than using the Alternative Data Envelopment Approach (ADEA) (Tahir et al., 2013).

Maredza et al. (2013) measure the changes between; efficiency and productivity at the period of 2000-2010 by using the (DEA) methodology. He argues that productivity was the main factor that fell to $17 \%$ lesser during crisis compared to the period prior to a crisis. On the other hand, another study was conducted in Malaysia to analysis the efficiency of seventeen IBs. Data was collected from the financial statements of 17 Islamic banks over the period of 2008-2009 using percentage analysis, descriptive statistics, and data envelopment analysis. It mainly concentrates on the IBs that have been divided into four categories, namely domestic bank-backed, stand-alone domestic, the foreign bank backed and the stand-alone foreign bank. The finding reveals that there is the inefficiency of profit as compared with cost and revenue. The different categories of Islamic banks IBs provided several efficiency scales which are necessary for IBs in Malaysia. This will provide for a better policy on activation of IBs in Malaysia (Rahim et al., 2015).

There a study conducted by Omari (2013) to reveal the measurement of efficiency performance in Jordanian Islamic banks using both methods of DEA and FRA during 2005-2009. The aim of the study was to measure and compare the development of a relative efficiency performance conducted between three of Islamic banks in Jordan, one of these banks was excluded from this study because it was recently established. This study found that Islamic banking is steady and efficient in terms of their inputs producing actual outputs.

In addition, Kuppusamy, Saleha \& Samudhram (2010) evaluated the Islamic banking performance measurement according to Shariah conformity. An analysis of Islamic banks' performance using the Maqasid-index by the implementation of seventeen profitability model sampling four banks namely Malaysia, Kuwait, Jordan, and Bahrain during the years 2001-2004. The developed model is called Shariah conformity and Profitability (SCNP) which assess financial performance indicators from conventional and Shariah. Shariah conformity is divided into numerous indicators for instance; Islamic income ratio, Islamic investment ratio, and profit sharing ratio. Furthermore, it can measure the bank profitability by gross profit ratio, return on equity (ROE), and return on asset (ROA) ratios. The finding asserts that most Islamic banks are able to reach high profitability and good Shariah compliance. But, there is just a bank with decreased level of profitability and Shariah 
compliance.

As shown by Mohammed \& Razak (2008) in Malaysia, they used the Maqasid-index to measure the performance of Islamic banking grounded on three general objectives that are; public interest, educating individuals and establishing justice. They used 1) Performance indicators. 2) The general Maqasid-Index. 3) Performance ratios. The best significance of the literature is that it proposes the objectives of IBs from the Maqasid Al-Shariah perspective. It also advanced the methodology that using this model has helped to generate the need to develop the performance measures dependent on Shariah's index. However, the result shows there are differences in the performances of selected Islamic banks and to achieving high performance in all the alternatives. Seven performance measurement ratios and performance indicators show inconsistency on the part of the individual Islamic banks to focusing on the overall Shariah objectives. However, this result has come at a suitable time for IBs to revisit their objectives after three decades of operations with more focusing on the general Shariah goals and reconsider their goals after three decades from its foundation (Ibrahim, 2015).

Again, Ngalim\& Ismail (2015) used the Islamic vision development based on indicators and analyses Islamic banks' performance among all Islamic banks in Indonesia, Malaysia, and GCC countries with selected samples. The objective is to propose an alternative measures that can have impact on Islamic bank activities not only towards achieving the objective of Shariah or Maqasid al Shariah's but also to give some guidance to Islamic banks on how they could improve their operations and focus in order to support the development of human well-being and economic growth without sacrificing their profitability-based objective as a vision by the Islam. (Islamic vision development based) IVBD Indicators were applied to Islamic banks in two regions involving Indonesia, Malaysia, and GCC nations. The results were as follows: first, the vision of Islamic bank as an institution that not only provides funding but, more importantly, play the role of a simulator for the development of both human and the economy is possible as proven by Indonesia Islamic Bank (1) IIB1. It is not only to provide a profit-sharing-based financing and move away from controversial contracts like Bay-Bi, ThaminAjil.

It possesses institutes to monitor and support its customers to ensure that their investment in them is secured. This provides hope to the global Muslim community to see a more promising rather than a failure of Islamic banking and finance in its socioeconomic obligations such as highlighted by Asutay (2008). Second is to ensure that Islamic banking and financial system works in an economy, educating both the public as potential customers as well as the people within the banking system that moving forward is crucial. It is also important to make sure that they are always reminded to be a better Muslim by continuous reminders via Islamic teachings. Islamic banks could and should include in these honest efforts and closely to free the Muslims from tied up in the conventional banking system. Though the authorities in each country do not obligate such effort, it is such an honor to know that Indonesia Islamic bank (1) IIB1, Malaysian Islamic Bank (7) MIB7, United Arab Emirate Islamic bank (5) UAEIB5 and United Arab Emirates (6) UAEIB 6 take it seriously. Third is to promote the right values and the right environment of a bank that operated based on Shariah is important to ensure that delivery of services could be executed in the best 
manner since identifying the right person is not easy at a stage of the recruitment. Despite that, banks should have a social and the charity work network to offer social responsibility to its community. As a fraction of their institution's social responsibility (CSR), nevertheless, establishing a commitment through continuous programs such as that of IIB1 and KSA Islamic Bank (1) KSAIB1 is exceptional. This only supports the notion that the more one gives, the more one will get benefits back. Other way shows how these banks really believe in the promises from the lawgiver. Fourth, non-financial performance measures such as, to gives more insights especially for Islamic banks since it is based on Shariah that aims overall, that real human well-being or (Falah) not only in this world but the hereafter. It is not important which bank supports or achieves the highest score, but more importantly, banks could have some guides on what they should be focusing on and learn from each other on how to fulfill their social and religious obligations. This empirical evidence reveals that the accurate position most of the Islamic banks are is not yet fulfilling the indicators of Maqasid for development. However, they are also not totally deviated from the 'intended' path of an anticipated Islamic bank as measured by the IVDB indicators. Achieving the ideal position of a Shariah financial institution requires a continuous improvement and these indicators are proposed to guide the path to the intended ideal state of an Islamic bank.

\section{Data and Methodology}

The main aim of this study is to compute the performance of three Islamic banks in Jordan by utilized; FRA, DEA, and MI over the period 2010-2013. Table 1 presents the 16 ratios of FRA which classified into four categories namely; profitability ratios, liquidity, leverage and activity (Samad et al, 1999; Samad\& Hassan, 2000).

Table 1: The Financial Ratio Analysis

\begin{tabular}{|l|l|}
\hline FRA & Ratios \\
\hline Profitability ratios & $\begin{array}{l}\text { EPS }=\text { (profit }- \text { dividends on preferred stock) } \\
\text { (outstanding share }) \\
\text { 2. ROA }=\text { (profit }- \text { profit } * \text { tax }) \div \text { total asset. } \\
\text { 3. } \mathrm{ROE}=\text { (profit }- \text { profit } * \text { tax }) \div \text { total equity. } \\
\text { 4. NIM }=\text { (profit and commissions income / total } \\
\text { income } * 100 \%) \\
\text { 5. CIM }=(\text { Credit profit } \div \text { credit facilities } * 100 \%) \\
\text { 6. ITTA }=(\text { Total income/ total assets } * 100 \%)\end{array}$ \\
\hline Leverage ratio & $\begin{array}{l}\text { ER }=\text { equity } \div \text { total assets } * 100 \% . \\
\text { 2. DR }=\text { total liabilities } / \text { total assets } * 100 \% . \\
\text { 3. ETD = Shareholders Equity / Total Deposits }\end{array}$ \\
\hline
\end{tabular}




\begin{tabular}{|c|c|}
\hline & $\begin{array}{l}* 100 \% . \\
\text { 1. } 4 . \text { DTA = Total Deposits } / \text { Total Assets } \% \text {. }\end{array}$ \\
\hline Activity ratios & $\begin{array}{l}\text { 1. NCFA }=\text { Net credit facilities/ Total assets } * 100 \% \text {. } \\
\text { 2. NCFD = Net credit facilities/ Total deposits* } \\
\text { 100\%. } \\
\text { 3. EC = Shareholders equity/Credit Facilities* } 100 \% \text {. }\end{array}$ \\
\hline Liquidity ratios & $\begin{array}{l}\text { 1. } \mathrm{QR}=(\mathrm{Cash}+\text { cash equivalents }+ \text { marketable } \\
\text { investments }+ \text { accounts receivables }) / \text { current } \\
\text { liabilities. } \\
\text { 2. CITD = (Cash }+ \text { Investments }) \div \text { total deposits } * \\
\text { 100\%. } \\
\text { 3. CTID = (cash }+ \text { trading investments }) / \text { total } \\
\text { deposits. }\end{array}$ \\
\hline
\end{tabular}

On the other hand, the DEA ratios can be classified as two classes which are inputs and outputs. Table 2 presents the DEA rations and correspondence formulas (Braglia et al., 2007).

Table 2: data envelopment analysis measurement

\begin{tabular}{|c|c|c|c|c|}
\hline $\begin{array}{l}\text { Catego } \\
\text { ry }\end{array}$ & & Ratios & Formula & Description \\
\hline Inputs & 3 & $\begin{array}{l}\text { Fixed } \\
\text { asset. } \\
\text { Deposi } \\
\text { t \& } \\
\text { short-t } \\
\text { erm } \\
\text { fund. } \\
\text { Equity } \\
\cdot \\
\text { Gener } \\
\text { al \& } \\
\text { admini } \\
\text { stratio } \\
\text { n expens }\end{array}$ & $\begin{array}{l}\text { Efficiency of DMU } J= \\
\text { Ej }=\frac{\text { weighted sum of } \text { outputs }}{\text { weighted sum of inputs }}= \\
=\frac{\sum_{i=1 \text { ai } Y n}^{n}}{\sum_{i=1 \beta \mathrm{i} X m}^{m}} \\
\text { Where: } \\
\boldsymbol{\alpha}_{\mathrm{k}} \text { : is the weight of output } \mathrm{K} \\
\boldsymbol{\beta}_{\mathrm{k}} \text { : is the weight of input } \mathrm{K} \\
\mathbf{Y}_{\mathrm{kj}} \text { amount of output } \mathrm{K} \text { from } \mathrm{DMU} \mathrm{j} \\
\mathbf{X}_{\mathrm{kj}} \text { : is the amount of input } \mathrm{K} \text { from }\end{array}$ & $\begin{array}{l}\text { The efficiency score is } \\
\text { often expressed as a } \\
\text { number between } 0 \% \text { and } \\
100 \% \text { or constrained to } \\
\text { the interval }(0,1)\} \text {. A } \\
\text { DMU with a score less } \\
\text { than } 100 \% \text { is viewed } \\
\text { inefficient relative to } \\
\text { others. The assessment of } \\
\text { the efficiency of each } \\
\text { DMU (e.g. a productive } \\
\text { unit) demands the } \\
\text { definition of a single and } \\
\text { common set of weights for } \\
\text { anindividual plant, } \\
\text { therefore presenting the }\end{array}$ \\
\hline
\end{tabular}




\begin{tabular}{|c|c|c|c|}
\hline & es. & DMU j & problem of how this set can \\
\hline $\begin{array}{l}\text { Output } \\
\text { S }\end{array}$ & $\begin{array}{l}\text { 7. Total } \\
\text { credit } \\
\text { faciliti } \\
\text { es } \\
\text { 8. Deposi } \\
\text { t \& } \\
\text { short-t } \\
\text { erm } \\
\text { fund. } \\
\text { Other } \\
\text { earnin } \\
\text { g } \\
\text { assets. }\end{array}$ & & $\begin{array}{l}\text { efficiency is made by the } \\
\text { decision-maker unit } \\
\text { (DMU) only if none of its } \\
\text { (Inputs - Outputs) is } \\
\text { improved without } \\
\text { deteriorating the inputs or } \\
\text { outputs. }\end{array}$ \\
\hline
\end{tabular}

Furthermore, the following Table3 presents the MI variables and ratios (Mohammed, Taib, Dzuljastri, 2008).

Table 3: MI Variables and Ratios

\begin{tabular}{|c|c|c|c|}
\hline Objectives (W) & $\begin{array}{l}\text { Objective } \\
\text { Weight }\end{array}$ & Ratio & $\begin{array}{l}\text { Ratio } \\
\text { weight }\end{array}$ \\
\hline \multirow[t]{4}{*}{$\begin{array}{l}\text { Education individual } \\
\text { Tahdzib Al-Fard }\end{array}$} & \multirow{4}{*}{$.30(\mathrm{~W} 1)$} & $\begin{array}{l}\text { R1 }{ }^{1} \text { : Education grant/total } \\
\text { expense. }\end{array}$ & $.24(\mathrm{E} 1)$ \\
\hline & & $\begin{array}{l}\mathrm{R} 1^{2} \text { : Research expense/total } \\
\text { expense }\end{array}$ & $.27(\mathrm{E} 2)$ \\
\hline & & $\begin{array}{l}\mathrm{R}^{3}{ }^{3} \text { : Training Expense/total } \\
\text { expense }\end{array}$ & $.26(\mathrm{E} 3)$ \\
\hline & & $\begin{array}{l}\mathrm{R}^{4} \text { : Publicity expense/total } \\
\text { expense }\end{array}$ & $.23(\mathrm{E} 4)$ \\
\hline $\begin{array}{l}\text { Establishing Justice } \\
\text { Iqomatul Al-Adl }\end{array}$ & $.41(\mathrm{~W} 2)$ & $\begin{array}{l}\mathrm{R}^{2} \text { : Mudharabah and } \\
\text { Musharakah Modes / total }\end{array}$ & $.32(\mathrm{E} 5)$ \\
\hline
\end{tabular}




\begin{tabular}{|c|c|c|c|}
\hline & & investment mode ${ }^{\mathrm{s}}$ & \\
\hline \multirow[t]{3}{*}{$\begin{array}{l}\text { Public Interest Jalb } \\
\text { Al-Maslahah }\end{array}$} & \multirow{3}{*}{$.29(\mathrm{~W} 3)$} & $\mathrm{R}^{1}$ : Net income/Total assets & $.33(\mathrm{E} 6)$ \\
\hline & & $\mathrm{R}^{2}:$ Zakah/Net Asset & .30 (E7) \\
\hline & & \multirow{2}{*}{$\begin{array}{l}\mathrm{R}^{3}: \text { Investment in real } \\
\text { economic sector/ Total } \\
\text { Investment }\end{array}$} & \multirow[t]{2}{*}{$.27(\mathrm{E} 8)$} \\
\hline Total & 1 & & \\
\hline
\end{tabular}

Mathematically, an evaluation each of Islamic banking Maqasid (containing dimension and performance ratios) may be constructed into the following equations:

1. Maqasid (Educated Individual)

$\mathrm{PI}(\mathrm{O} 1)=\mathrm{W} 1_{\mathrm{x}} E 1_{\mathrm{x}}^{\mathrm{R} 1^{1}}+\mathrm{W} 1_{\mathrm{x}}^{\mathrm{E} 2} \mathrm{x}^{\mathrm{R} 1^{2}}+\mathrm{W} 1_{\mathrm{x}} 3_{\mathrm{x}}^{\mathrm{R} 1^{3}}+\mathrm{W} 1 \mathrm{x}^{\mathrm{E} 4}$ $\mathrm{x}^{\mathrm{R} 1^{4}}$

2. Maqasid (Justice)

$\mathrm{PI}(\mathrm{O} 2)=\mathrm{W} 2 \quad \mathrm{xE} 5 \times \mathrm{R} 2^{2}$

3. Maqasid (Maslahah)

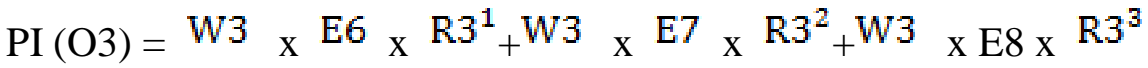

Thus, MI can be computed using the following formula:

$\mathrm{MI}=\mathrm{PI}(\mathrm{O} 1)+\mathrm{PI}(\mathrm{O} 2)+\mathrm{PI}(\mathrm{O} 3)$

\section{Result and Discussion}

This chapter investigates Jordanian Islamic banks efficiency in terms of their capacity to provide outputs with minimal inputs consumption. The analysis part consists of three sections: financial ratios analysis, data envelopment analysis model and MaqasidS hariah index.

\subsection{Financial Ratio Analysis (FRA)}

Profitability, liquidity, leverage, and activity ratios were compute of Jordanian Islamic banks over the period 2010-2013. 
Table 4: Financial Ratios Analysis for Jordan Islamic Bank Finance and Investment over the Period 2010-2013.

\begin{tabular}{|l|l|l|l|l|l|l|l|l|l|l|l|l|l|l|l|l|}
\hline JIBF \\
I
\end{tabular}


Table 5: Financial Ratios Analysis for Islamic International Arab Bank over the Period 2010-2013.

\begin{tabular}{|c|c|c|c|c|c|c|c|c|c|c|c|c|c|c|c|c|}
\hline IIAB & Pro & fita & ility & atio & & & & erag & rati & & Acti & ity ra & & & $\begin{array}{l}\text { lidity } \\
\text { OS }\end{array}$ & \\
\hline years & $\begin{array}{l}\mathbf{E} \\
\mathbf{P} \\
\mathbf{S}\end{array}$ & $\begin{array}{l}\mathbf{R} \\
\mathbf{O} \\
\mathbf{A}\end{array}$ & $\begin{array}{l}\mathbf{R} \\
\mathbf{O} \\
\mathbf{E}\end{array}$ & $\begin{array}{l}\text { NI } \\
\mathbf{M}\end{array}$ & $\begin{array}{l}\text { CI } \\
\mathbf{M}\end{array}$ & $\begin{array}{l}\text { IT } \\
\text { TA }\end{array}$ & $\begin{array}{l}\mathbf{E} \\
\mathbf{R}\end{array}$ & $\begin{array}{l}\mathbf{E} \\
\mathbf{T} \\
\mathbf{D}\end{array}$ & $\begin{array}{l}\mathbf{D} \\
\mathbf{R}\end{array}$ & $\begin{array}{l}\mathbf{D} \\
\mathbf{T} \\
\mathbf{A}\end{array}$ & $\begin{array}{l}\text { NC } \\
\text { FA }\end{array}$ & $\begin{array}{l}\text { NC } \\
\text { FD }\end{array}$ & $\begin{array}{l}\mathbf{E} \\
\mathbf{C}\end{array}$ & $\begin{array}{l}\mathbf{Q} \\
\mathbf{R}\end{array}$ & $\begin{array}{l}\text { CI } \\
\text { TD }\end{array}$ & $\begin{array}{l}\mathbf{C} \\
\mathbf{T I} \\
\mathbf{D}\end{array}$ \\
\hline 2010 & $\begin{array}{l}.0 \\
7\end{array}$ & $\begin{array}{l}.6 \\
4\end{array}$ & $\begin{array}{l}8.0 \\
2\end{array}$ & $\begin{array}{l}18 . \\
61\end{array}$ & $\begin{array}{l}1 . \\
69\end{array}$ & $\begin{array}{l}2.3 \\
4\end{array}$ & $\begin{array}{l}7 . \\
97\end{array}$ & $\begin{array}{l}22 . \\
21\end{array}$ & $\begin{array}{l}92 . \\
03\end{array}$ & $\begin{array}{l}35 . \\
91\end{array}$ & $\begin{array}{l}74 . \\
56\end{array}$ & $\begin{array}{l}207 \\
.63\end{array}$ & $\begin{array}{l}10 . \\
69\end{array}$ & $\begin{array}{l}.2 \\
7\end{array}$ & $\begin{array}{l}29 . \\
04\end{array}$ & .27 \\
\hline 2011 & $\begin{array}{l}.1 \\
1\end{array}$ & $\begin{array}{l}.9 \\
4\end{array}$ & $\begin{array}{l}11 . \\
81\end{array}$ & $\begin{array}{l}23 . \\
05\end{array}$ & $\begin{array}{l}2 . \\
17\end{array}$ & $\begin{array}{l}3.0 \\
5\end{array}$ & $\begin{array}{l}7 . \\
99\end{array}$ & $\begin{array}{l}25 . \\
6\end{array}$ & $\begin{array}{l}92 . \\
01\end{array}$ & $\begin{array}{l}31 . \\
23\end{array}$ & $\begin{array}{l}69 . \\
75\end{array}$ & $\begin{array}{l}223 \\
.34\end{array}$ & $\begin{array}{l}11 . \\
46\end{array}$ & $\begin{array}{l}.2 \\
2\end{array}$ & $\begin{array}{l}27 . \\
18\end{array}$ & .22 \\
\hline 2012 & $\begin{array}{l}.1 \\
2\end{array}$ & $\begin{array}{l}9 \\
8\end{array}$ & $\begin{array}{l}11 . \\
44\end{array}$ & $\begin{array}{l}35 . \\
54\end{array}$ & $\begin{array}{l}3 . \\
38\end{array}$ & $\begin{array}{l}4.0 \\
6\end{array}$ & $\begin{array}{l}8 . \\
60\end{array}$ & $\begin{array}{l}24 . \\
03\end{array}$ & $\begin{array}{l}91 . \\
4\end{array}$ & $\begin{array}{l}35 . \\
80\end{array}$ & $\begin{array}{l}63 . \\
85\end{array}$ & $\begin{array}{l}178 \\
.34\end{array}$ & $\begin{array}{l}13 . \\
47\end{array}$ & $\begin{array}{l}.2 \\
6\end{array}$ & $\begin{array}{l}28 \\
34\end{array}$ & .26 \\
\hline 2013 & $\begin{array}{l}.1 \\
6\end{array}$ & $\begin{array}{l}1 . \\
1 \\
7\end{array}$ & $\begin{array}{l}13 . \\
47\end{array}$ & $\begin{array}{l}46 . \\
65\end{array}$ & $\begin{array}{l}3 . \\
98\end{array}$ & $\begin{array}{l}4.1 \\
1\end{array}$ & $\begin{array}{l}8 . \\
67\end{array}$ & $\begin{array}{l}24 . \\
56\end{array}$ & $\begin{array}{l}91 . \\
33\end{array}$ & $\begin{array}{l}35 . \\
32\end{array}$ & $\begin{array}{l}65 . \\
19\end{array}$ & $\begin{array}{c}184 \\
.55\end{array}$ & $\begin{array}{l}13 . \\
31\end{array}$ & $\begin{array}{l}.2 \\
1\end{array}$ & $\begin{array}{l}22 . \\
35\end{array}$ & .21 \\
\hline Mean & $\begin{array}{l}0 . \\
11\end{array}$ & $\begin{array}{l}0 . \\
9 \\
3\end{array}$ & $\begin{array}{l}11 . \\
18\end{array}$ & $\begin{array}{l}30 . \\
96\end{array}$ & $\begin{array}{l}2 . \\
80\end{array}$ & $\begin{array}{l}3.3 \\
9\end{array}$ & $\begin{array}{l}8 . \\
31\end{array}$ & $\begin{array}{c}24 . \\
10\end{array}$ & $\begin{array}{l}91 . \\
69\end{array}$ & $\begin{array}{l}34 . \\
57\end{array}$ & $\begin{array}{l}68 . \\
34\end{array}$ & $\begin{array}{l}198 \\
.46\end{array}$ & $\begin{array}{l}12 . \\
23\end{array}$ & $\begin{array}{l}0 . \\
24\end{array}$ & $\begin{array}{l}26 . \\
73\end{array}$ & $\begin{array}{l}0.2 \\
4\end{array}$ \\
\hline
\end{tabular}


Table 6: Financial Ratios for Jordan Dubai Islamic Bank over the Period 2010-2013.

\begin{tabular}{|c|c|c|c|c|c|c|c|c|c|c|c|c|c|c|c|c|}
\hline \multirow{2}{*}{$\begin{array}{c}\text { JDIB } \\
\text { years }\end{array}$} & \multicolumn{6}{|c|}{ Profitability ratios } & \multicolumn{4}{|c|}{ Leverage ratios } & \multicolumn{3}{|c|}{$\begin{array}{l}\text { Activity } \\
\text { ratio }\end{array}$} & \multicolumn{3}{|c|}{$\begin{array}{l}\text { Liquidity } \\
\text { ratios }\end{array}$} \\
\hline & $\begin{array}{l}\text { EP } \\
S\end{array}$ & $\begin{array}{l}\text { RO } \\
\text { A }\end{array}$ & $\begin{array}{l}\mathbf{R} \\
\mathbf{O} \\
\mathbf{E}\end{array}$ & $\begin{array}{l}\text { NI } \\
\text { M }\end{array}$ & $\begin{array}{l}\mathbf{C} \\
\mathbf{I} \\
\mathbf{M}\end{array}$ & $\begin{array}{l}\mathbf{I} \\
\mathbf{T} \\
\mathbf{T} \\
\mathbf{A}\end{array}$ & $\begin{array}{l}\mathbf{E} \\
\mathbf{R}\end{array}$ & $\begin{array}{l}\text { ET } \\
\text { D }\end{array}$ & $\begin{array}{l}\mathbf{D} \\
\mathbf{R}\end{array}$ & $\begin{array}{l}\mathbf{D} \\
\mathbf{T} \\
\mathbf{A}\end{array}$ & $\begin{array}{l}\mathbf{N} \\
\mathbf{C F} \\
\mathbf{A}\end{array}$ & $\begin{array}{l}\mathbf{N} \\
\mathbf{C} \\
\mathbf{F} \\
\mathbf{D}\end{array}$ & $\begin{array}{l}\mathbf{E} \\
\mathbf{C}\end{array}$ & $\begin{array}{l}\mathbf{Q} \\
\mathbf{R}\end{array}$ & $\begin{array}{l}\text { C } \\
\text { I } \\
\text { T } \\
\text { D }\end{array}$ & $\begin{array}{l}\text { C } \\
\text { T } \\
\text { I } \\
\text { D }\end{array}$ \\
\hline 2010 & $\begin{array}{l}-.0 \\
5\end{array}$ & $\begin{array}{l}-1.4 \\
8\end{array}$ & $\begin{array}{l}-3 \\
60\end{array}$ & $\begin{array}{l}17 \\
46\end{array}$ & $\begin{array}{l}1 . \\
7 \\
7\end{array}$ & $\begin{array}{l}2 . \\
0 \\
0\end{array}$ & $\begin{array}{l}40 . \\
98\end{array}$ & $\begin{array}{l}27 \\
8.7 \\
3\end{array}$ & $\begin{array}{l}59 . \\
02\end{array}$ & $\begin{array}{l}1 \\
4 . \\
7 \\
0\end{array}$ & $\begin{array}{l}43 . \\
41\end{array}$ & $\begin{array}{l}2 \\
9 \\
5 . \\
2 \\
2\end{array}$ & $\begin{array}{l}94 . \\
41\end{array}$ & $\begin{array}{l}1.5 \\
6\end{array}$ & $\begin{array}{l}1 . \\
7 \\
4\end{array}$ & $\begin{array}{l}1 . \\
5 \\
6\end{array}$ \\
\hline 2011 & .06 & $\begin{array}{l}1.5 \\
3\end{array}$ & $\begin{array}{l}4.6 \\
5\end{array}$ & $\begin{array}{l}33 \\
26\end{array}$ & $\begin{array}{l}4 . \\
5 \\
0\end{array}$ & $\begin{array}{l}5 . \\
6 \\
3\end{array}$ & $\begin{array}{l}32 . \\
83\end{array}$ & $\begin{array}{l}22 \\
3.9 \\
7\end{array}$ & $\begin{array}{l}67 . \\
17\end{array}$ & $\begin{array}{l}1 \\
4 . \\
6 \\
6\end{array}$ & $\begin{array}{l}55 . \\
99\end{array}$ & $\begin{array}{l}3 \\
8 \\
1 . \\
9 \\
4\end{array}$ & $\begin{array}{l}58 . \\
64\end{array}$ & $\begin{array}{l}0.8 \\
0\end{array}$ & $\begin{array}{l}0 . \\
9 \\
9\end{array}$ & $\begin{array}{l}0 . \\
8 \\
0\end{array}$ \\
\hline 2012 & .02 & .45 & $\begin{array}{l}1.6 \\
8\end{array}$ & $\begin{array}{l}50 \\
57\end{array}$ & $\begin{array}{l}5 . \\
3 \\
3\end{array}$ & $\begin{array}{l}3 . \\
7 \\
0\end{array}$ & $\begin{array}{l}26 . \\
74\end{array}$ & $\begin{array}{l}18 \\
5.0 \\
6\end{array}$ & $\begin{array}{l}73 . \\
26\end{array}$ & $\begin{array}{l}1 \\
4 . \\
4 \\
5\end{array}$ & $\begin{array}{l}55 . \\
55\end{array}$ & $\begin{array}{l}3 \\
8 \\
4 . \\
4 \\
7\end{array}$ & $\begin{array}{l}48 . \\
13\end{array}$ & $\begin{array}{l}0.8 \\
2\end{array}$ & $\begin{array}{l}1 . \\
1 \\
3\end{array}$ & $\begin{array}{l}.8 \\
2\end{array}$ \\
\hline 2013 & .01 & $\begin{array}{l}0.2 \\
8\end{array}$ & $\begin{array}{l}1.1 \\
4\end{array}$ & $\begin{array}{l}40 \\
13\end{array}$ & $\begin{array}{l}7 . \\
1 \\
4\end{array}$ & $\begin{array}{l}4 . \\
2 \\
3\end{array}$ & $\begin{array}{l}24 . \\
47\end{array}$ & $\begin{array}{l}17 \\
4.2 \\
0\end{array}$ & $\begin{array}{l}75 . \\
53\end{array}$ & $\begin{array}{l}1 \\
4 . \\
0 \\
5\end{array}$ & $\begin{array}{l}44 . \\
88\end{array}$ & $\begin{array}{l}3 \\
1 \\
9 . \\
5 \\
0\end{array}$ & $\begin{array}{l}54 . \\
52\end{array}$ & $\begin{array}{l}1.5 \\
7\end{array}$ & $\begin{array}{l}1 . \\
5 \\
9\end{array}$ & $\begin{array}{l}1 . \\
5 \\
7\end{array}$ \\
\hline Mean & .01 & .19 & .97 & $\begin{array}{l}35 \\
36\end{array}$ & $\begin{array}{l}4 . \\
6 \\
8\end{array}$ & $\begin{array}{l}3 . \\
8 \\
9\end{array}$ & $\begin{array}{l}31 . \\
26\end{array}$ & $\begin{array}{l}21 \\
5.4 \\
9\end{array}$ & $\begin{array}{l}68 . \\
74\end{array}$ & $\begin{array}{l}1 \\
4 . \\
4 \\
6\end{array}$ & $\begin{array}{l}49 . \\
96\end{array}$ & $\begin{array}{l}3 \\
4 \\
5 . \\
2 \\
8\end{array}$ & $\begin{array}{l}63 . \\
93\end{array}$ & $\begin{array}{l}1.1 \\
9\end{array}$ & $\begin{array}{l}1 . \\
3 \\
6\end{array}$ & $\begin{array}{l}1 . \\
1 \\
9\end{array}$ \\
\hline
\end{tabular}

The Tables; 4, 5, and 6 clarifies the financial ratios for Jordanian Islamic banks that used in measuring bank's efficiency. 


\section{Macrothink}

SPSS version 20.0 was used to analyze means and descriptive analysis of the total FRA data that collected from the Jordanian Islamic banks. Regarding to descriptive analysis of total FRA in Table 7; JDIB recorded the best FRA mean (52.3725), followed by IIAB with 42.5 as FRA mean, and lastly JIBFI recorded the lowest FRA mean (29.6356). On the other hand, the standard deviation of JDIB (95.06) is the highest comparing with IIAB (51.4), and the lowest is JIBFI (34.1). Thus, the Ratios of JDIB was changed frequently more than the ratios of other two banks.

Table 7: Descriptive Analysis of Total FRA

\begin{tabular}{|l|l|l|l|l|l|}
\hline & $\mathrm{N}$ & $\begin{array}{l}\text { Minimu } \\
\mathrm{m}\end{array}$ & $\begin{array}{l}\text { Maximu } \\
\mathrm{m}\end{array}$ & Mean & $\begin{array}{l}\text { Std. } \\
\text { Deviation }\end{array}$ \\
\hline JIBFI & 16 & .31 & 92.50 & 29.6356 & 34.14409 \\
IIAB & 16 & .11 & 198.46 & 42.5 & 51.47582 \\
JDIB & 16 & .01 & 345.28 & 52.3725 & 95.06338 \\
$\begin{array}{l}\text { Valid N (list } \\
\text { wise) }\end{array}$ & 16 & & & & \\
\hline
\end{tabular}

Based on the total FRA means of three Jordanian Islamic banks, JIBFI considered as the lowest ratio rank, IIAB is the second ratio rank, and JDIB the highest ratio rank 8.

Table 8: Total FRA Means Ranking of Jordanian Islamic Banks

\begin{tabular}{|l|l|l|l|}
\hline NO. & Banks Name & Mean & Ranking \\
\hline 1 & JIBFI & 29.63 & 3 \\
\hline 2 & IIAB & 42.5 & 2 \\
\hline 3 & JDIB & 52.37 & 1 \\
\hline
\end{tabular}

\subsection{Data Envelopment Analysis}

The Efficiency Measurement System (EMS), special software is utilized to investigate the efficiency of Jordanian Islamic banks. In sensible terms, the DEA is performed for every year separately. Table 9 display the DEA results based on the model with two outputs and four inputs. 
Table 9: Jordanian Islamic Banks Efficiency Scores over the Period 2010-2013.

\begin{tabular}{|l|l|l|l|l|}
\hline Years & IIAB efficiency & JIBFI efficiency & JDIB efficiency & Mean \\
\hline $\mathbf{2 0 1 3}$ & $99.91 \%$ & $67.33 \%$ & $82.76 \%$ & $83.33 \%$ \\
\hline $\mathbf{2 0 1 2}$ & $97.42 \%$ & $78.01 \%$ & $83.37 \%$ & $86.27 \%$ \\
\hline $\mathbf{2 0 1 1}$ & $53.40 \%$ & $33.76 \%$ & $88.32 \%$ & $58.49 \%$ \\
\hline $\mathbf{2 0 1 0}$ & $53.27 \%$ & $38.54 \%$ & $48.86 \%$ & $46.89 \%$ \\
\hline Mean & $0.76 \%$ & $0.544109 \%$ & $0.758289 \%$ & \\
\hline
\end{tabular}

The table 9 shows that IIAB improves its situation from the inefficient score of $53.27 \%$ in 2010 to be efficient a score of $99.91 \%$ in 2013. On the other hand, the JIBFI has randomly improved over the period, and the lowest efficiency score of 33.76\% was in 2011 and the results from total credit facilities and lower bank outputs. Also, shows that JIBFI improves its situation to be efficient a score of $78.01 \%$ in 2012 . However, shows that JDIB improves its situation from the inefficient score of $48.86 \%$ in 2010 to be efficient a score of $88.32 \%$ in 2011. Generally, the improvement is obvious in Jordanian Islamic banks industry from $46.89 \%$ in 2010 reaching to $83.33 \%$ in 2013 . The CRS efficiency is significantly higher, on average, for IIAB compared to JIBFI by around $22 \%$ percentage points. Overall, for IIAB compared with JDIB was slightly change that not exceed 0.5 percentage.

The following table shows the ranked of Islamic banks in Jordan depending on efficiency scores means over the period 2010-2013.

Table 10: Ranked of Jordanian Islamic Banks Efficiency Scores.

\begin{tabular}{|l|l|l|l|}
\hline No. & Bank Name & Efficiency score & Ranking \\
\hline 1 & JIBFI & $0.544 \%$ & 3 \\
\hline 2 & IIAB & $0.76 \%$ & 1 \\
\hline 3 & JDIB & $0.758 \%$ & 2 \\
\hline
\end{tabular}

\subsection{The Enhancements and Slack Analysis}

One of the purposes of this study is to provide suggestions to improve the efficiency of Islamic banks in Jordan. This class explains how the JIBFI, IIAB, and JDIB can increase their efficiency scores. This class examines the slacks analytical of the variables (inputs-outputs) of the inefficient Islamic banks. The slacks are the modification of variables (inputs-outputs) that ought to be eliminated to the Decision Making Units (DMU) under evaluation to be 
considered as efficient. Slacks ordinarily refer to the inputs and the distinction between the target and actual inputs with outputs values.

The findings reports of a slack analysis for Jordanian Islamic banks as the following;

Table 11: Slacks for IIAB

\begin{tabular}{|l|l|l|l|l|l|l|}
\hline IIAB & \multicolumn{2}{|l|}{ Inputs } & \multicolumn{2}{l|}{ Outputs } \\
\hline Year & $\begin{array}{l}\text { Deposit } \\
\text { and short } \\
\text { term } \\
\text { funding }\end{array}$ & $\begin{array}{l}\text { Fixed } \\
\text { assets }\end{array}$ & $\begin{array}{l}\text { Administr } \\
\text { ation and } \\
\text { general } \\
\text { expenses }\end{array}$ & Equity & $\begin{array}{l}\text { Total } \\
\text { facilities }\end{array}$ & $\begin{array}{l}\text { Other } \\
\text { earning } \\
\text { assets }\end{array}$ \\
\hline 2010 & 364042358 & 10969161 & 11173782 & 0 & 0 & 22453601 \\
\hline 2011 & 328844613 & 12101561 & 13254723 & 0 & 0 & 30049581 \\
\hline 2012 & 11602140 & 499206 & 509996 & 2671220 & 0 & 0 \\
\hline 2013 & 0 & 0 & 0 & 0 & 0 & 0 \\
\hline
\end{tabular}

Table 12: slacks for JIBFI

\begin{tabular}{|l|l|l|l|l|l|l|}
\hline JIBFI & \multicolumn{2}{|l|}{ Inputs } & \multicolumn{2}{l|}{ Outputs } \\
\hline Year & $\begin{array}{l}\text { Deposit } \\
\text { and short } \\
\text { term } \\
\text { funding }\end{array}$ & $\begin{array}{l}\text { Fixed } \\
\text { assets }\end{array}$ & $\begin{array}{l}\text { Administr } \\
\text { ation and } \\
\text { general } \\
\text { expenses }\end{array}$ & Equity & $\begin{array}{l}\text { Total } \\
\text { facilities }\end{array}$ & $\begin{array}{l}\text { Other } \\
\text { earning } \\
\text { assets }\end{array}$ \\
\hline 2010 & $\begin{array}{l}373886068 \\
4\end{array}$ & 61427315 & 48773784 & 0 & 0 & 13076472 \\
411 & $\begin{array}{l}517035119 \\
8\end{array}$ & 86097109 & 74467003 & 0 & 0 & $\begin{array}{l}18581225 \\
9\end{array}$ \\
\hline 2012 & 768431336 & 0 & 12449665 & 64494389 & 0 & 0 \\
\hline 2013 & $\begin{array}{l}143532397 \\
3\end{array}$ & 28292067 & 24162849 & 0 & 86434520 & 8 \\
\hline
\end{tabular}


Table 13: Slacks for JDIB

\begin{tabular}{|l|l|l|l|l|l|l|}
\hline JDIB & \multicolumn{2}{|l|}{ Inputs } & \multicolumn{2}{l|}{ Outputs } \\
\hline Year & $\begin{array}{l}\text { Deposit } \\
\text { and short } \\
\text { term } \\
\text { funding }\end{array}$ & $\begin{array}{l}\text { Fixed } \\
\text { assets } \\
\text { ation and } \\
\text { general } \\
\text { expenses }\end{array}$ & Administr & Equity & $\begin{array}{l}\text { Total } \\
\text { facilities }\end{array}$ & $\begin{array}{l}\text { Other } \\
\text { earning } \\
\text { assets }\end{array}$ \\
\hline 2010 & 43199098 & 9642315 & 9867834 & 0 & 12181513 & 4142600 \\
\hline 2011 & 7382634 & 1429938 & 1234493 & 0 & 25942770 & 0 \\
\hline 2012 & 17147272 & 2524107 & 0 & 25341389 & 0 & 0 \\
\hline 2013 & 18595608 & 3352001 & 3328418 & 0 & 49393129 & 0 \\
\hline
\end{tabular}

\subsection{Maqasid Index (MI)}

MI approach model in this study conducted on Jordanian Islamic banking industries that have the highest assets. There are three Islamic banks in Jordan. As the following;

Table 14: Development of Islamic Banking Assets in Jordan

\begin{tabular}{|l|l|l|l|l|}
\hline Name of Banks & 2010 & 2011 & 2012 & 2013 \\
\hline JIBFI & 2603683927 & 2898300754 & 2898300754 & 3020492066 \\
\hline IIAB & 1096162778 & 1127482501 & 1173775421 & 1348244028 \\
\hline JDIB & 268088438 & 350398767 & 475217035 & 528462073 \\
\hline
\end{tabular}

Sources: adapted from the CBJ website.

Table 14 above clarifies that the assets of JIBFI and IIAB have positive growth by $2-15 \%$ year by year. On the other hand, the assets of JDIB have a positive highest growth by $11-35 \%$ year by year. The increases in banks assets of the industry provide an indication of the increases in the banks' performance. Anyway, the indications don't seem to be sufficient and unable to represent a comprehensive performance consistent with its main purpose of Maqasid index.

\subsubsection{Maqasid Index (MI) Performance Ratio of Jordanian Islamic Banks}

Firstly, the MI that utilizes to calculate the performance of Islamic banks industry in Jordan 
depending on the determined ratio. As mentioned in chapter three that MI taken in this study is three performance ratios; first one education individuals Tahdzib Al-Fard, secondly Justice Iqomatul Al-Adle, and third one public interest Jalb Al-Maslahah. The following table 15 the performance ratios to MI of Jordanian Islamic banks.

Table 15: MI Performance Ratio of Jordanian Islamic banks 2010-2013 (in percentage)

\begin{tabular}{|c|c|c|c|c|c|c|c|c|}
\hline \multirow[t]{2}{*}{ Banks } & \multicolumn{4}{|l|}{$\mathrm{MI} 1^{\mathrm{st}}$} & MI $2^{\text {nd }}$ & \multicolumn{3}{|l|}{$\mathrm{MI} 3^{\text {rd }}$} \\
\hline & $\mathrm{R} 1^{1}$ & $\mathrm{R} 2^{1}$ & $\mathrm{R} 3^{1}$ & $\mathrm{R} 4^{1}$ & $\mathrm{R} 2^{2}$ & $\mathrm{R} 3^{1}$ & $\mathrm{R} 3^{2}$ & $\mathrm{R} 3^{3}$ \\
\hline JIBFI & .299 & .108 & .288 & 2.048 & 46.767 & 1.077 & .006 & 15.333 \\
\hline IIAB & .423 & .174 & .376 & 2.462 & 16.228 & 0.947 & .029 & 63.545 \\
\hline JDIB & .050 & .041 & .774 & 3.637 & 34.206 & .339 & 0.017 & 60.107 \\
\hline
\end{tabular}

Table 15 shows three objectives of Maqasid Index Performance Ratio (MIPR) as the following;

1. MIPR of the first objective education/ Tahdzib Al-Fard.

A. Education Grants / Total Expenses $\left(\mathrm{R}^{1}\right)$

Depend on the assessment of MIPR Regarding expenditure on Human Resources (HR) in the form of education grant from Jordanian Islamic banks industry; IIAB has the highest value of .423. Then, following by JIBFI and JDIB by .299 and .050 respectively. These results states that IIAB provides higher value funding allocation for education sector $\mathrm{R}^{1}$ comparing with JIBFI and JDIB in Jordan.

B. Research Expenses / Total Expenses $\left(\mathrm{R}^{2}\right)$

MIPR as a first aim relating to research expenditure present the budget for Islamic banks industry development. Accordingly, in 2010-2013 IIAB has the highest performance ratio comparing with other Jordanian Islamic banks by .174\%. Following by JIBFI .108\%, this is because IIAB routine to implement program called professional training and scientific research. Meanwhile, JDIB is still limited to spending more costs or research it's just by $.041 \%$ compared with other Islamic banks in Jordan. This means need to allocate more funds in development and research due to it help the sustainability and progress of banks.

C. Training Expense / Total Expenses $\left(\mathrm{R}^{3}\right)$

The training cost intended MIPR is the budget assigned for the education program and training so as to improve the necessary activities of banks industry. According to this ratio, JDIB ranked the highest by $.774 \%$ compared with other banks industry. The second rank is IIAB by $.376 \%$. Then, JIBFI ranked the lowest by .288\%.

D. publicity / total expenses $\left(\mathrm{R}^{4}\right)$ 


\section{Macrothink}

The fourth MIPR as a first aim is publication cost that conducted to improve the banks industry. This activity over the period 2010-2013, JDIB ranked the highest publication cost by $3.637 \%$ compared with other bank industry. Second rank IIAB by $2.462 \%$. Then, JIBFI has the lowest in their expenditure for publication cost by $2.048 \%$. The ratio publication in Jordanian Islamic banks is relatively low because to make the society has awareness on Islamic banks need continuous efforts to introduce and educate people so that they can manage finance wisely to improve the quality of living in the future with stay away of dealing with conventional banks. The lack of information received by the society is due to lack of education and socialization of Islamic banks in Jordan.

2. Maqasid index performance ratio $2^{\text {nd }}$ goal Al-Adle (Justice)

\section{A. Mudharabah and Musharakah Modes / total investment modes $\left(\mathrm{R}^{2}\right)$}

at the second goal of MIPR regarding Islamic banks performance in allocating their activities for AL-Adle reason, this study choose one ratio about how big is financing ratio that conducted depend on profit sharing Mudharabah and cooperation Musharakah scheme / aqad. Financing activities those scheme / aqad, JIBFI has ranked the highest by $46.767 \%$ of their financing is implemented with Mudharabah and Musharakah scheme. Then,followed by JDIB which rank $34.206 \%$. Meanwhile, IIAB ranked as lowest one with $16.228 \%$. This result clarifies that, JIBFI industry development has a better than other Jordanian Islamic bank depend on financing products.

3. Maqasid-Index Performance Ratio $3^{\text {rd }}$ goal Public Interest (Jalb Al-Maslahah)

A. Net Income / Total assets $\left(\mathrm{R}^{1}\right)$

In MIPR, the third objective regarding Jalb Al-Maslahah is becoming the main element in the Islamic finance system. It can also mean the extent to which the existence of Islamic banking industry can be felt it benefits; shareholders, stakeholders, and the society.

The first ratio from this aim is could be seen from the profit ratio of Islamic banking industry. Depend on this ratio; JIBFI has a higher profitability over the past 4 years by $1.077 \%$ compared to others. Following by; IIAB and JDIB ratio $0.947 \%$ and $.339 \%$ respectively. In the MI framework, this ratio classified as the Hifzhul Maal aspect since Islamic banking has tried to manage and use their wealth optimally and prudentially. This also could be present that when a financial institution has not produced a maximum "profit" then there is not income redistribution to the stakeholders.

b. Zakah / net income $\left(\mathrm{R}^{2}\right)$

As for allocation of Zakah funds channeled by the Islamic banking industry is as their social activities. Depend on the facts over the year 2010-2013, this data for fund allocation of Jordanian Islamic banks Zakah. Gave negative results with lesser values as for allocation of Zakah funds channeled by the Jordanian Islamic banks' industry is as their social activities. From the last table show that IIAB has the highest value by $.029 \%$. Following by; JDIB and JIBFI by $.017 \%$ and $.006 \%$ respectively. 
c. investment in real sector / total investment $\left(\mathrm{R}^{3}\right)$

The third performance ratio looks at how much investment banking in favor of the real sector of the total investment made. The previous table clarifies that IIAB has a higher ratio of $63.545 \%$. Following by; JDIB and JIBFI by $60.107 \%$ and $15.333 \%$ respectively. Its means that investment almost entirely not touches real sector and a very few allocated on the non-real sector. This clarifies that the level of partisanship and the orientation of Islamic banking in real sector in Jordan is lower.

\subsection{Health Level of Jordanian Islamic Banks under the Performance Indicators}

The following table 999 is the rank result that has been adapted with weight from Mohammad and Taib (2008) of three Jordanian Islamic banks are used as the aim of research. Based on these weights, as for Maqasid index on the first goal, JIBFI is as the largest Jordanian Islamic banks ranked as highest on publicity expenses in the performance process of Islamic banking. The second objective of Maqasid index JIBFI ranked the highest performance compared to two other banks. While third objective of Maqasid index IIAB, ranked the highest performance that improving welfare ranked as the highest performance compared with two other banks.

Table 16: Ranked of Three Islamic Banks in Jordan Depend on Performance Indicators (PI).

\begin{tabular}{|c|c|c|c|c|c|c|c|c|c|c|}
\hline \multirow{2}{*}{$\begin{array}{l}\text { Bank } \\
\text { s }\end{array}$} & \multicolumn{5}{|c|}{$1^{\text {st }}$ objective PI } & \multirow{2}{*}{$\begin{array}{l}2^{\text {nd }} \\
\text { Objecti } \\
\text { ve PI } \\
\mathrm{R}_{2}{ }^{1}\end{array}$} & \multicolumn{4}{|c|}{$3^{\text {rd }}$ Objective PI } \\
\hline & $\mathrm{R}_{1}^{1}$ & $\mathrm{R}_{1}^{2}$ & $\mathrm{R}_{1}{ }^{3}$ & $\mathrm{R}_{1}^{4}$ & Total & & $\mathrm{R}_{3}{ }^{1}$ & $\mathrm{R}_{3}{ }^{2}$ & $\mathrm{R}_{3}{ }^{3}$ & Total \\
\hline JIBF & .0002 & .0000 & .0002 & .0014 & .0613 & .001030 & .0010 & .0000 & .0120 & .0130 \\
\hline I & 15 & 87 & 24 & 13 & 58 & & 30 & 05 & 05 & 42 \\
\hline IIAB & $\begin{array}{l}.0003 \\
04\end{array}$ & $\begin{array}{l}.0001 \\
40\end{array}$ & $\begin{array}{l}.0002 \\
93\end{array}$ & $\begin{array}{l}.0016 \\
98\end{array}$ & $\begin{array}{l}.0212 \\
91\end{array}$ & .000906 & $\begin{array}{l}.0009 \\
06\end{array}$ & $\begin{array}{l}.0000 \\
25\end{array}$ & $\begin{array}{l}.0497 \\
55\end{array}$ & $\begin{array}{l}.0506 \\
87\end{array}$ \\
\hline JDIB & $\begin{array}{l}.0000 \\
36\end{array}$ & $\begin{array}{l}.0000 \\
33\end{array}$ & $\begin{array}{l}.0006 \\
03\end{array}$ & $\begin{array}{l}.0025 \\
09\end{array}$ & $\begin{array}{l}.0448 \\
78\end{array}$ & .000324 & $\begin{array}{l}.0003 \\
24\end{array}$ & $\begin{array}{l}.0000 \\
14\end{array}$ & $\begin{array}{l}.0470 \\
63\end{array}$ & $\begin{array}{l}.0474 \\
03\end{array}$ \\
\hline
\end{tabular}


4.6. Jordanian Islamic Banks Health Level based on Maqasid index

Table 17: Rank of Jordanian Islamic Banks Depend on Maqasid Index

\begin{tabular}{|l|l|l|l|l|l|l|}
\hline No. & Banks & PI O1 & PI O2 & PI O3 & $\begin{array}{l}\text { MI } \\
{\left[\begin{array}{lll}\text { PI O1+PI O2+PI } \\
\text { O3] }\end{array}\right.}\end{array}$ & $\begin{array}{l}\text { Ranke } \\
\mathrm{d}\end{array}$ \\
\hline 1 & JIBFI & .061358 & .001030 & .013042 & 0.07543 & 2 \\
\hline 2 & IIAB & .021291 & .000906 & .050687 & 0.072884 & 3 \\
\hline 3 & JDIB & .044878 & .000324 & .047403 & 0.092605 & 1 \\
\hline
\end{tabular}

The last table presents the rank of banks depend on Maqasid index; JDIB the highest rank following by JIBFI then IIAB.

Based on three tables ranking; 8,10 , and 17 , the ranking total of three measurements methods of JDIB is 4, IIAB is 6 , and JIBFI is 8 . Here, the lowest ranking totals represent the best performance ranking. Thus, JDIB considers as the best performance ranking followed by IIAB and lastly JIBFI. Table 18 shows the Jordanian Islamic banks performance according to integration between various measurements tools.

Table 18: Integration between Measurements Tools.

\begin{tabular}{|l|l|l|l|l|l|l|}
\hline No. & Bank & FRA & DEA & MI & Total & Overall Rank \\
\hline 1 & JIBFI & 3 & 3 & 2 & 8 & 3 \\
\hline 2 & IIAB & 2 & 1 & 3 & 6 & 2 \\
\hline 3 & JDIB & 1 & 2 & 1 & 4 & 1 \\
\hline
\end{tabular}

\section{Conclusion and Future Works}

This study focuses on enhance the performance measurements of Jordanian Islamic banks. The previous studies were utilized one from three measurements methods (FRA, DEA, and MI). There was limitation in the studies that integrate between more than one measurement tools to measure the performance of Jordanian Islamic banks. This study conduct the performance measurements based on integration between FRA, DEA, and MI tools. The 
proposed integration between these methods was utilized to compute and rank performance of the three Jordanian Islamic banks; JIBFI, IIAB, and JDIB. The significance results show the best performance rank is JDIB, followed by IIAB, and lastly JIBFI. The contribution of this study is the utilizing of several performance measurement methods which produce accurate measurement criteria more than utilizing one or two methods. In future the proposed measurement criteria could be enhanced through formulate more accurate calculation based on the combination of measurements methods (e.g. identify the coefficient importance of each method rather than give the same importance for all methods). On the other hand, the performance of Islamic banks from various countries could be measured using the proposed criteria in order to assure the usefulness of the combination of measurement tools.

\section{References}

Al-Naimat, S. 2013."The Central Bank of Jordan and Its Relation with Islamic Banks In Jordan". International Journal of Business and Management.Vol. 8 (9).

Antonio, Mohd S.,Sanrego Y.\&Taufiq M. 2012. "An Analysis of Islamic Banking Performance: Maqashid Index Implementation In Indonesia and Jordan". Journal of Islamic Finance. Vol.1 (1), 2012: 012-029.

AssiAmarh Mohammed Yahya. 2010. "Assess the financial performance of Islamicbanks: An Empirical Study on the Jordan Islamic Bank for Finance and Investment".Mktatbatalkhatib. p.1-135.

Asutay, Mehmet. 2008. "Islamic banking and finance: social failure". New Horizon 169, p. 10 -12 .

Bhattacharyya, S. \& Antony, J. P. 2010."Measuring organizational performance and organizational excellence of SMEs-Part 2: an empirical study on SMEs in India".Measuring Business Excellence, 14(3). p. 42-52.s

Braglia, M., S. Zanoni\& L. Zavonella. 2007. "Measuring and Benchmarking Productive Systems Performances Using DEA an Industrial Case Production Planning and Control". Vol. 14.No. 6.p.542-554.

Hamouri, B., Hamouri, Q. \&Radaydeh, M. 2014. "The Impact of Islamic Financing on Companies' Performance (A Comparative Study of Industrial Companies Listed on theAmman Stock Exchange). "International Journal of Business and social Science.Vol.5(7).June4.

Al-Talib, G, S. 2005."Assessing the financial performance of the Islamic Bank". Dar Al-salamm.Dar Al-Salaam for printing and publishing.p. 12-23.

Hunjra, A. I., \& Bashir, A. 2014."Comparative Financial Performance Analysis ofConventional and Islamic Banks in Pakistan". Bulletin of Business and Economics (BBE), 3(4).p. 196-206.

Ibrahim, M. 2015. "Measuring the Financial Performance of Islamic Banks".Journal of 
Applied Finance and Banking. Vol. 5.No. 3.p. 93-104.

Johnes, J. Izzeldin, M. \& Pappas, V. 2012."A comparison of Performance of Islamic and Conventional Banks". Journal of Applied Finance \& Banking, 2(3). p. 123-161.

Johnes, J., Izzeldin, M., \& Pappas, V. 2009. Efficiency in Islamic and conventional banks: A comparison based on financial ratios and data envelopmentanalysis. EconomicsWorkingPaper Series, The Economics Department, Lancaster University.

Johnes, J., Izzeldin, M., \& Pappas, V. 2009. Efficiency in Islamic and conventional banks: A comparison based on financial ratios and data envelopmentanalysis. Economics Working Paper Series, The Economics Department,Lancaster University.

Kuppusamy, Mudiarasan, Saleha, Ali Salman danSamudhram, Ananda. 2010. "Measurement of Islamic Banks Performance Using a Shariah Conformity and ProfitablityModel". Jurnal Review of Islamic Economics, 13 (2): p. 35-48.

Kurd A, A. 2010. "Islamic banksobjectives and services". Electronic encyclopaedia. Vol. 3.Egypt: Azhar University.

Majid, A. Mariani., S. David, S. \&Battisti. 2005. "Efficiency in Islamic andConventional Banking". Journal of productivity analysis,3(4). p. 25-43.

Maredza, A., \&Ikhide, S. 2013. "Measuring the impact of the global financial crisis on efficiency and productivity of the banking system in South Africa". Mediterranean Journal of Social Sciences, 4(6). p.553.

Mohamed, M. O., Razak, D., \&MdTaib, F. 2008. "The Performance Measures of Islamic Banking Based on the Maqasid Framework".

Mohammed, DzuljastridanTaib. 2008. The Performance Measures of Islamic Banking Based on the Maqashid Framework. Paper of IIUM International AccountingConference (INTAC IV) held at Putra Jaya Marriott.

Najjar, N. J. 2013. "Can financial ratios reliably measure the performance of banks inbahrain".International Journal of Economics and Finance, 5(3).p.152-163.

Ngalim, S. M., \& Ismail, A. G. 2015. An Islamic Vision Development Based Indicators in Analyzing the Islamic Banks Performance: Evidence from Malaysia, Indonesia and selected GCC Countries (No. 1436-2).

Omari, H. O., Moh'd M, A. \& 2013. "Performance efficiency of the Jordanian IslamicBanks using data envelopment analysis and financial ratios analysis". European Scientific Journal. p. 1-68.

Rahim, S. R. M., Bakar, J. b. A. \&ApGanapathy, T. 2015. "How efficient are Islamic banksin Malaysia". Journal of Business Studies Quarterly, 6(3). p. 164-174.

Samad, A. Hassan, M. K. 2000. "the performance of Malaysia islamic banks during 1984-1997. An explanatory study".Thoughts on economics. 10 (1-2). 1-25. 


\section{Macrothink}

Samad, A., \& Hassan, M. 1999. "The performance of Malaysian Islamic bank during 1984-1997: An exploratory study". International Journal of Islamic Financial Services.1(3).p.1-14

Shehzad, F., Sehrish, S., Saleem, F., Yasir, M., \& Ahmed, K. 2012."Financial performance analysis of Islamic banks and conventional banks in pakistan: A comparative study Interdisciplinary". Journal of Contemporary Research in Business, 4(5). p. 186-200.

Tahir, I. M., Razali, A. R., \&Haron, S. 2013. "Analysing Islamic Bank Efficiency in Malaysia Using the Standard and Alternative Approaches to Data EnvelopmentAnalysis". Journal of Economics and Behavioral Studies, 5(11), 798.

\section{Websites}

(CBJ) central bank of Jordan, website: (www.cbj.gov.jo/index.php).

\section{Copyright Disclaimer}

Copyright for this article is retained by the author(s), with first publication rights granted to the journal.

This is an open-access article distributed under the terms and conditions of the Creative Commons Attribution license (http://creativecommons.org/licenses/by/3.0/). 